NOTA CRÍTICA

\title{
Los mercados del turismo comunitario en América Latina Perspectivas para una agenda de inVestigación
}

\author{
ERnest Cañada \\ Centro de Investigación Alba Sud \\ Escuela Universitaria de Turismo y Hotelería \\ Universidad de Barcelona
}

\section{Resumen}

Este artículo tiene como objetivo identificar los términos de debate, reflejados en la literatura académica, en torno a los distintos tipos de mercado a los cuales acceden las iniciativas de turismo comunitario y qué implicaciones pueden derivarse de diferentes estrategias comerciales. Con una presentación concisa de la problemática, y de los retos que están en juego, se pretende abrir la discusión y proponer una agenda de investigación que ayude a escalar el análisis en términos más significativos para el propio sector comunitario.

Palabras clave: Comercialización, desarrollo rural, turismo comunitario, turismo doméstico.

\section{Community-based tourism markets in Latin America \\ Perspectives for a Research Agenda}

\begin{abstract}
This article aims to identify the terms of debate reflected in academic literature, around the different types of market to which community tourism initiatives have access. It is also focused on the implications that can derive from different commercial strategies. With a concise presentation of this problem and its challenges, the aim is to start the discussion and propose a research agenda that will help improve up the analysis in terms that are more significant for the community sector itself.
\end{abstract}

Keywords: Commercialization, rural development, community-based tourism, domestic tourism

Fecha de recepción: 27 de mayo de 2019. Fecha de aceptación: 23 de junio de 2019

CÓMO CITAR: Cañada, E. (2019). Los mercados del turismo comunitario en América Latina Perspectivas para una agenda de investigación. Dimensiones Turísticas, 3(5), 96-105. https://doi.org/10.47557/KNYB9816 


\section{E. Cañada}

D urante las tres últimas décadas, se han multiplicado iniciativas de turismo comunitario diversas. En América Latina la profusión de este tipo de experiencias ha sido especialmente notable. A la par, se han multiplicado los procesos de acompañamiento externo con la participación de actores de todo tipo, tanto públicos como privados, lo cual ha dado mayor peso y protagonismo al sector. Del mismo modo, el turismo comunitario se ha convertido en uno de los grandes temas de investigación en las ciencias sociales, hasta tal punto que resulta difícil estar al día de todo lo que se publica y, a su vez, poder valorar los aportes reales de la investigación disponible.

Además de una producción incesante, uno de los primeros problemas para poder desbrozar qué pueden aportar los distintos estudios realizados es que no siempre está claro si realmente todos ellos hacen referencia a experiencias de turismo comunitario o no, aunque se presenten bajo esa etiqueta. En ocasiones se toman en cuenta iniciativas que, en realidad, tienen muy poco que ver con una gestión colectiva o comunitaria, y se trata simplemente de turismo ubicado en un entorno rural. Esta mezcla de experiencias de distinta índole obstaculiza llegar a conclusiones relevantes. Otra de las dificultades en el análisis de la literatura es la acumulación de estudios de caso meramente descriptivos, sin anclaje suficiente en debates reales, surgidos de las preocupaciones de los propios actores, o de marcos teóricos que permitan entender procesos complejos con resultados sociales dispares (Gascón y Milano, 2017). Una complicación añadida tiene que ver con la diversidad en las formas de organización de las comunidades a lo largo de América Latina, y esto se traduce también en una pluralidad de expresiones del turismo comunitario que dificulta en ocasiones el reconocimiento mutuo, cuando no deriva en debates en su mayoría estériles planteados en términos de legitimidad. Asimismo, la discusión se complejiza cuando se entiende que no es necesaria la existencia de organización colectiva previa para que pueda desarrollarse turismo comunitario, sino que en el propio desarrollo de la actividad turística pueden recrearse formas de organización y gestión comunitarias (Ruiz-Ballesteros, 2017a; Ruiz-Ballesteros y Cáceres-Feria, 2016).

En este contexto, ante la enorme cantidad de literatura existente sobre el turismo comunitario y las distintas dificultades identificadas, parece necesario tratar de poner cierto orden en su análisis en torno a algunos debates fundamentales, que prioritariamente deberían surgir de las preocupaciones expresadas por el propio sector comunitario. Es urgente identificar temas trascendentes y profundizar en ellos de forma segmentada para avanzar hacia nuevas síntesis de conocimiento que orienten próximos trabajos de investigación.

A efectos del presente artículo ${ }^{1}$ se toma como referencia de turismo comunitario aquellas perspectivas que enfatizan la idea de gestión de base local (Cabanilla, 2018), gestión basada en el control colectivo por parte de la población local sobre el conjunto de la actividad (Cañada, 2013, 2014) o una organización basada en la participación local colectiva

${ }^{1}$ Este artículo se elaboró en el marco del proyecto Fortalecer el criterio de inclusividad en el turismo responsable: una respuesta a los retos de la Educación para la Justicia Global, ejecutado por Alba Sud con el apoyo del Ayuntamiento de Barcelona a través del Programa de Educación para la Justicia Global (convocatoria 2018). Quiero hacer constar mi agradecimiento a Luna Reinoso y Marta Salvador, del equipo de Alba Sud, por sus aportes al artículo. 
en el desarrollo, gestión y repartición de beneficios del turismo, asentada en la propiedad y control de la propia actividad, e integrado en la economía local (Ruiz-Ballesteros, 2017b; Ruiz-Ballesteros, Hernández, Coca, Cantero y Del Campo, 2008). Se enfatiza esta visión del turismo comunitario como forma de gestionar y/o organizar estas actividades a partir de la colectividad organizada con voluntad de control, frente a otras aproximaciones que han puesto más el acento en una oferta basada en lo rural o en la mera participación de la población oferente.

\section{Objetivos y metodología}

Este texto tiene como objetivo identificar los términos de debate, reflejados en la literatura académica, en torno a una de las cuestiones centrales en el análisis del turismo comunitario: el acceso a los mercados de las iniciativas comunitarias y qué implicaciones pueden derivarse de las distintas estrategias comerciales escogidas. Durante años, la capacidad de comercialización ha sido uno de los temas clave en la discusión sobre la viabilidad de este tipo de propuestas de gestión colectiva. Sin embargo, los términos en los que fue planteada esta discusión fueron muy parciales, se puse más énfasis en el fracaso que en lo que funcionaba, y no ayudaron a entender las dinámicas comerciales en las que se estaban viendo involucradas muchas iniciativas comunitarias que lograron consolidarse como estructuras empresariales de gestión colectiva. En este sentido, el artículo propone una revisión de la discusión y una aproximación a los nuevos retos a los que se enfrenta el turismo comunitario en relación con el acceso a los mercados, definidos en términos plurales expresamente.

La preocupación por esta cuestión surge a partir del acompañamiento a redes e iniciativas de turismo comunitario en lugares de América Latina por más de quince años. Responde a un criterio subjetivo de valoración de lo que se considera más relevante en los debates y preocupaciones del propio sector comunitario. Con la presentación concisa de la problemática y de los retos que están en juego, se pretende abrir la discusión y proponer una agenda de investigación que ayude a escalar el análisis en términos más significativos para el propio sector comunitario.

\section{El cuestionamiento de la capacidad comercial del turismo comunitario}

Desde hace años, uno de los temas centrales en el análisis del turismo comunitario ha sido la escasa capacidad comercial y de generación de ingresos por este tipo de emprendimientos una vez puestos en marcha, lo cual derivaría en su posterior abandono. Esto se atribuye en gran medida a las dificultades para acceder al mercado y consolidar una demanda que les permita vender determinados servicios, hacer viables empresarialmente sus iniciativas y mejorar sus condiciones de vida (Ashley y Goodwin, 2007; Bartholo y Bursztyn, 2012; García y Font, 2013; Mitchell y Muckosy, 2008). También se ha argumentado en términos 


\section{E. Cañada}

autocríticos, desde alguna de las grandes instituciones internacionales de cooperación al desarrollo que impulsaron en sus inicios el turismo comunitario, que las estimaciones y expectativas en torno a la comercialización de muchas iniciativas a los que se había apoyado no fueron realistas (Denman, 2001).

En este sentido, desde una perspectiva global, no meramente circunscrita a América Latina, y desde la literatura académica, se han señalado múltiples causas, no necesariamente coincidentes, que ayudarían a explicar esta baja capacidad de comercialización: parte de las dificultades para consolidar proyectos exitosos tenían que ver con la falta de experiencia, conocimiento y habilidades en turismo entre los residentes locales, convertidas en barreras culturales que les habría hecho necesitar ayuda externa para superarlas (Kamarudin, 2013; López-Guzmán, Sánchez-Cañizares y Pavón, 2011; Tolkach y King, 2015); este apoyo externo no siempre tuvo la suficiente continuidad para consolidar procesos (Tolkach y King, 2015). El trabajo de acompañamiento externo se centró más en la construcción de infraestructuras y capacitación técnica en un sector no tradicional que en la construcción de vínculos con el mercado (Ashley y Goodwin, 2007; Ashley y Roe, 2002); hubo un fuerte desconocimiento del funcionamiento del mercado y de los encadenamiento con los sectores más dinámicos de la industria turística (Armstrong, 2011; Mitchell y Muckosy, 2008); a las iniciativas comunitarias les costaba darse a conocer, promocionarse y comercializar los proyectos a nivel internacional (Timothy y White, 1999; Sakata y Prideaux, 2013); existían problemas de accesibilidad física y de comodidad en las iniciativas comunitarias, como carreteras, electricidad, agua potable, que limitaban el acceso y permanencia de los turistas (Harwood, 2010; Timothy y White, 1999); sus productos estaban escasamente diversificados, lo cual incrementaba su vulnerabilidad ante cualquier imprevisto o cambio en las orientaciones del mercado (Harwood, 2010); la dependencia con respecto a donantes externos dificultaba los procesos de consolidación comercial de este tipo de iniciativas (Hamzah y Mohamad 2012; Pawson, Arcy y Richardson, 2016; Zapata, 2011); los niveles de fracaso han sido más elevados cuando las iniciativas han tratado de vender en el mercado internacional, cuyo funcionamiento les resulta extraño, que cuando han ofrecido servicios turísticos directos a la población local con quienes comparten un mismo sustrato cultural (Zapata et al., 2011); una parte del acompañamiento a través de los organismos de cooperación internacional, autoridades públicas y organismos multilaterales, especialmente los que han tenido acceso a fondos financieros más importantes, han priorizado una orientación hacia el mercado de altos ingresos de carácter internacional, desatendiendo otros mercados con más potencialidades para algunas de esas experiencias (Cañada, 2013; Zapata, 2011).

Con base en algunos de estos argumentos, que sin duda han podido identificar una problemática real en iniciativas del sector, hubo quien puso en cuestión la misma viabilidad del turismo comunitario. De ahí que una parte de la literatura académica se concentrara en tratar de poner de manifiesto otras vías para reducir la pobreza, incrementando el vínculo de sectores con menos recursos con las dinámicas del capital turístico tradicional. Dentro de estos nuevos esquemas podía incorporarse el turismo comunitario, pero se ensalzaban 
sobre todo otras formas de relación con el sector empresarial hegemónico. Dentro de este nuevo esquema destacaron estrategias como el pro-poor tourism, formulado por primera vez por Loitte Touche en 1999 en una publicación del Departamento para el Desarrollo Internacional (DFID) (Harrison y Schipani, 2007), e impulsado por diversos académicos que desarrollaron su carrera profesional en Gran Bretaña (Ashley y Roe, 2002; Ashley, Roe y Goodwin, 2001; Goodwin, 2013). En una versión modificada, a partir del enfoque de negocios inclusivos desarrollada por la cooperación técnica holandesa, este nexo potencial entre "pobres" y capital turístico fue institucionalizado por la Organización Mundial del Turismo (омт) a través de su programa ST-EP (OMT, 2004, 2006; SNV y OMT, 2010). Estas nuevas estrategias resultaban más compatibles con posiciones favorables a la liberalización de los mercados y el rol de las grandes empresas capitalistas en los países del Sur Global. Sin embargo, las críticas a estas perspectivas señalaron su incapacidad real para reducir la pobreza cuando, en realidad, el gran capital turístico se beneficiaba de diferenciales de renta globales y eran responsables de mayores niveles de empobrecimiento, desigualdad y vulnerabilidad de las poblaciones rurales, además de acusar a este tipo de propuestas de estar al servicio de un intento de legitimar el papel de los grandes capitales turísticos cada vez más puestos en cuestión (Cicci e Hidalgo, 2013; Harrison, 2008; Gascón, 2015, 2017).

A pesar de la extensión de este debate, la focalización de los estudios en el fracaso de los procesos comerciales del turismo comunitario y el desarrollo de nuevas estrategias que facilitaran la expansión y legitimación del capital turístico, e incluso el necesario contrapunto crítico, invisibilizaron una parte de los procesos de comercialización exitosos que muchas otras iniciativas comunitarias pudieron consolidar a través de diferentes vías.

\section{Mercados diversos por vías de acceso múltiples}

A pesar del fracaso comercial de muchas iniciativas de turismo comunitario, hasta cierto punto magnificadas en comparación con lo que ocurre con muchos otros negocios en el mercado convencional, una parte de este sector ha logrado acceder al mercado y consolidarse empresarialmente. Tanto los mercados como las estrategias seguidas han sido plurales.

Una parte del turismo comunitario en América Latina, igual que muchas otras pequeñas y medianas iniciativas familiares, se ha orientado hacia el mercado doméstico, próximo geográficamente y anclado en una oferta de servicios para clases medias y bajas. A pesar de la menor atención por parte de las políticas públicas y agencias de cooperación internacional, este tipo de iniciativas se ha asentado sobre la capacidad de inversión local y un avance progresivo orientado a partir de la propia experiencia y aprendizaje, facilitada por compartir idioma y referentes culturales entre quienes ofrecen sus servicios y quienes los demandan (Zapata et al., 2011). Durante la década pasada, ciertas mejoras en las economías de algunos países de la región permitieron a sectores de clases trabajadoras ampliar su capacidad de consumo en ocio. Es el caso, por ejemplo, del desarrollo del valle de Huatanay, convertido desde los años noventa en el principal espacio de recreación de las 


\section{E. Cañada}

clases medias de Cuzco, Perú, a partir de pequeños negocios gastronómicos articulados con base en una identidad territorial (Asensio, 2017; Asensio y Trivelli, 2011). Otro caso significativo, es el de la Finca de los Hermanos Cerrato en la Reserva Natural de El Tisey en Estelí, Nicaragua. La iniciativa, gestionada por nueve unidades familiares y con una fuerte orientación hacia la investigación en agricultura orgánica, se convirtió en espacio de recreación durante los fines de semanas para familias del departamento de Estelí o de la capital, Managua, a unas dos horas en vehículo propio, y con un fuerte reclamo por la gastronomía tradicional (Cañada, 2014). Su caso es también indicativo de la mayor resiliencia de este tipo de negocios orientados hacia mercados locales. A raíz de la crisis política y de vulneración de derechos que sufre Nicaragua desde abril de 2018, su turismo internacional ha decaído dramáticamente. En estos términos, muchas de las iniciativas de turismo comunitario basadas en el mercado internacional han tenido que cerrar operaciones. En cambio, proyectos como la Finca de los Hermanos Cerrato, u otras similares, han logrado mantenerse, a pesar de las dificultades. En El Salvador, también destaca la experiencia de la Cooperativa Los Pinos, en el municipio El Congo, a orillas del lago Coatepeque, a menos de 45 minutos desde San Salvador. En este caso la base del negocio es también un restaurante y un mirador, pero su particularidad es que, además de clases medias locales, también atrae un segmento de turistas salvadoreños residentes en Estados Unidos que regresan por vacaciones al país de origen. Gracias a un volumen de visitación muy destacado, más de doce mil personas en 2018, el turismo se ha convertido en un mercado muy favorable para la venta directa de café en bolsas ya tostado o en taza (Cañada, 2017a). En estos casos los procesos de comercialización se han construido de forma directa, a través de boca-oreja, publicidad en medios locales y la colaboración de medios de comunicación del propio país. Su consolidación no ha requerido de otros intermediarios comerciales.

En el acceso al mercado internacional por parte de iniciativas de turismo comunitario han operado dos vías. Por una parte, algunas experiencias han logrado especializarse en un segmento del mercado internacional, especialmente el de turismo mochilero, a través de su posicionamiento en algunas guías de viaje, como Lonely Planet, o, cada vez más, prescriptores en Internet. En algunos casos también se han visto favorecidos por una ubicación geográfica estratégica, en rutas del turismo internacional bien posicionadas. Este podría ser el caso de la Finca Magdalena, una cooperativa ubicada en la isla Ometepe de Nicaragua. Su aparición en guías internacionales la convirtió en un lugar de paso privilegiado en la visita de miles de jóvenes de distintas partes del mundo que querían ascender al volcán Maderas. Sin embargo, el grueso del acceso al mercado internacional ha requerido procesos de profesionalización de la gestión comercial y la creación o vinculación con estructuras intermediarias de tour-operación que garantizaran unas llegadas de clientes regulares. Durante años, las redes nacionales de turismo comunitario que establecieron en distintos países de América Latina, trataron de impulsar empresas tour-operadoras propias, organizadas en diversas formas (Cañada, 2015). Tal vez el caso más desarrollado de este tipo de procesos, hasta su fallida en 2018, fuera el de la asociación ACTUAR en Costa Rica (Cañada, 2017b). Sin embargo, el papel de tour-operadoras privadas locales, 
con conocimiento del territorio y de la oferta comunitaria han jugado un papel clave para garantizar este florecimiento comercial. En algunos casos hay un compromiso auténtico con las comunidades y la mejora de su bienestar, que se traduce no solo en los procesos de comercialización, sino también en capacitación técnica o mejora de los productos, además de una gestión transparente con las comunidades. Experiencias como Origins en Salta, Argentina, Localista en San José, Costa Rica, o Totonal en Playa del Carmen, México, son solo algunos ejemplos de este tipo de iniciativas. Pero también hay tour-operadoras tradicionales que se han visto en la necesidad de ampliar y adaptar su oferta a partir de las demandas de sus clientes, y que no muestran ningún compromiso ni interés real por las comunidades rurales.

El papel de las tour-operadoras locales no puede entenderse sin hacer mención a agencias y plataformas, que son las que mayoritariamente captan comercialmente a los clientes en los países del Norte Global. En la relación comercial entre agencias europeas y norteamericanas y las tour-operadoras locales latinoamericanas destaca el hecho que la relación con el cliente final la conserva de forma predominante la agencia, quien no publicita quienes son sus corresponsales en destino y gestiona directamente con el cliente todas las variables de la venta. Ejemplos relevantes podrían ser Double Sens, Human Trip - La Route des Voyages de Francia, América Andina o Napo Tours de Alemania, Beyond Tourism de Inglaterra, Emotion Planet de Bélgica o Pasion Terre de Canadá. Por su parte, las plataformas en Internet, con un creciente peso en el mercado de viajes, a partir de una amplia oferta en su Web, ponen en contacto directo a los turistas con las tour-operadoras locales y se desentienden de la gestión concreta del viaje, lo que les permite reducir sus comisiones en comparación con las agencias tradicionales, lo cual deja un mayor margen a las tour-operadoras locales. Este tipo de oferta se adapta mejor a las tendencias del consumo y en muy poco tiempo ha conseguido hacerse un hueco muy destacado en este mercado. Ejemplos destacados de este tipo de plataformas podrían ser Better Places de Holanda, Crooked Trails, Visit.org o Lokal Travel de Estados Unidos, Fairaway o Trip Me de Alemania o Babel Voyages de Francia. Tanto en unos casos como en otros, las estrategias de márquetin y de comercialización no tienden a ofertar viajes en exclusiva de turismo comunitario, más bien se incluye oferta comunitaria, más o menos extensa, en itinerarios muy diversificados, que tampoco huyen de reclamos tradicionales. Habitualmente tampoco se menciona al turismo comunitario en la oferta, más bien se vincula la propuesta a la posibilidad de disfrutar de entornos naturales, de autenticidad o de contacto con la gente local. Se estructura así una clara diferencia entre la gestión operativa de la comunidad de los referentes comunicativos.

\section{Conclusiones}

La consolidación de distintos mercados para el turismo comunitario tiene múltiples implicaciones. La primera es a todas luces evidente, lejos de la desaparición que le auguraban sus críticos, se ha afianzado una oferta de turismo comunitario amplia. Sin duda ha ha- 


\section{E. Cañada}

bido fracasos y los seguirá habiendo, pero no parece que no haya lugar para este tipo de iniciativas. Segunda, el turismo doméstico adquiere una importancia central para muchas comunidades, y permite dinámicas mucho más resilientes, tanto a las dinámicas sociales, como a los procesos de cambio climático y encarecimiento del precio de los combustibles a causa del peak oil que se avecina en un futuro inmediato. A pesar de la falta de atención, tanto por parte de las autoridades públicas como de la investigación académica, en los mercados de proximidad se están produciendo transformaciones sustanciales para el futuro del turismo y del turismo comunitario en particular. Tercero, la eclosión de una creciente oferta internacional que integra turismo comunitario no podría entenderse sin los cambios experimentados en un mercado turístico en el que coexisten estructuras fordistas y postfordistas. La ampliación de un segmento de mercado interesado en experiencias "singulares" y "auténticas", alejadas de la estandarización de la oferta tradicional (Maccannell, 2003), ha abierto una gama de posibilidades cada vez más amplia, que puede ir desde el interés por visitar destinos que desaparecerán con el cambio climático, el turismo gastronómico, el dark tourism o la misma oferta gestionada por organizaciones comunitarias. Cuarta, este crecimiento de la demanda y de las estructuras de intermediación conIleva nuevos retos y riesgos a las comunidades que apuesten por insertarte en el mercado internacional. El decaimiento de las redes nacionales de turismo comunitario en distintos lugares de América Latina, demasiado dependientes de la cooperación externa y de intereses particulares, ha dejado paso a que las tour-operadoras privadas acabaran jugando un papel cada vez más relevante en el acompañamiento externo a las comunidades. Todo esto suscita nuevos interrogantes sobre las implicaciones que acabarán contrayendo. En definitiva, la comercialización del turismo comunitario, lejos de convertirse en el factor letal que ponía en cuestión su viabilidad, se abre a una pluralidad de caminos. Desde el ámbito de la investigación social se debería tratar de entender mejor cuáles son los procesos en curso y, quienes se sientan comprometidos con la organización comunitaria, ayudar a identificar riesgos y opciones que favorezcan un desarrollo más equitativo, inclusivo y sostenible.

\section{Fuentes citadas}

Armstrong, R. L. (2011). An analysis of the conditions for success of community-based tourism enterprises. Leeds: Leeds Metropolitan University.

Asensio, R. H. (2017). Los nuevos incas. La economía política del desarrollo rural andino en Quispicanchi (2000-2010). Lina: Instituto de Estudios Peruanos (IEP).

Asensio, R. H. y Trivelli, C. (2011). Crecimiento económico, cohesión social y trayectorias divergentes Valle Sur-Ocongate (Cuzco-Perú). Santiago de Chile: Rimisp, Documento de Trabajo núm. 65, Programa Dinámicas Territoriales Rurales.

Ashley, C. y Roe, R. (2002). Working with the private sector on pro-poor-tourism. Londres: ODIIIED. 
Ashley, C., Roe, D. y Goodwin, H. (2001). Pro-poor tourism strategies: Making tourism work for the poor. A review of experience. Nottingham: ODI, IIED, CRT.

Ashley, C. y Goodwin, H. (2007). Turismo pro-pobre. ¿Qué ha ido bien y qué ha ido mal? Londres: oDı, Opinión, núm. 80.

Bartholo, R. y Bursztyn, I. (2012). O processo de comercialização do turismo de base comunitária no Brasil: Desafios, potencialidades e perspectivas. Sustentabilidade em Debate, 3(1), 97-116.

Cabanilla, E. (2018). Turismo comunitario en América Latina, un concepto en construcción. Siembre, 5(1), 121-131.

Cañada, E. (2013). Turismo en Centroamérica. Un diagnóstico para el debate. Managua: Enlace.

Cañada, E. (2014). Turismo comunitario en Centroamérica. Experiencias y aprendizajes. Managua: Editorial Enlace.

Cañada, E. (2015). La comercialización del turismo comunitario en América Latina. Anuario de Estudios Centroamericanos, 41, 159-189.

Cañada, E. (2017a). Contribución del turismo comunitario a la economía campesina: la 111 Cooperativa Los Pinos en El Salvador. En J. Gascón y C. Milano. El turismo en el mundo rural. ¿Ruina o consolidación de las sociedades campesinas e indígenas? (pp. 111-137). Tenerife: Pasos.

Cañada, E. (2017). Estructuras de intermediación turística procomunitarias. La experiencia comercial de Actuar en Costa Rica. Gazeta de Antropología, 33(1).

Cicci, D. M. e Hidalgo, M. (2013). Turismo y alivio de la pobreza: fundamentos teóricos y evidencias empíricas. En J. Gascón, S. Morales y J. Tresserras (Eds.). Cooperación en turismo: nuevos desafíos, nuevos debates (pp. 407-427). Barcelona: FTR.

Denman, R. (2001). Directrices para el desarrollo del turismo comunitario. Gland: WWF Internacional.

García, V. y Font, X. (2013). Community based tourism: critical success factors. ICRT Occasional Paper.

Gascón, J. (2015). Pro-poor tourism as a strategy to fight rural poverty: A Critique. Journal of Agrarian Change, 15(4), 499-518.

Gascón, J. (2017). Pro-Poor Tourism. En L. Lowry (Ed.). The SAGE international encyclopedia of travel and tourism (pp. 974-976). Thousand Oaks: SAGE Publications.

Gascón, J. y Milano, C. (2017). El turismo en el mundo rural. ¿Ruina o consolidación de las sociedades campesinas e indígenas? Tenerife: Pasos.

Goodwin, H. (2013). Turismo y reducción de la pobreza. En J. Gascón, S. Morales y J. Tresserras (eds.). Cooperación en turismo: nuevos desafíos, nuevos debates (pp. 387405). Barcelona: FTR.

Hamzah, A. y Mohamad, N. H. (2012). Critical Success Factors of Community Based Ecotourism: Case Study of Miso Walaihomestay, Kinabatangan, Sabah. Malaysian Forester, 75(1), 29-42.

Harrison, D. (2008). Pro-poor Tourism: a critique. Third World Quarterly, 29(5), 851-868.

Harrison, D. y Schipani, S. (2007). Lao tourism and poverty alleviation: Community-based tourism and the private sector. Current Issues in Tourism, 10(2-3), 194-230.

Harwood, S. (2010). Planning for community based tourism in a remote location. Sustaina- 


\section{E. Cañada}

bility, 2, 1909-1923.

Kamarudin, K. H. (2013). Local institutional development and transformation through community based rural tourism: Investigation of three communities in East Coast of Malaysia. 4th Workshop and Meeting Rural Research and Planning Group (RRPG), Rural Transformation and Institutional Development. Institut Teknologi Bandung, Indonesia, September 9-10, 2013.

López-Guzmán, T., Sánchez-Cañizares, S. y Pavón, V. (2011). Community-based tourism in developing countries: A case study. Tourismos: An International Multidisciplinary Journal of Tourism, 6(1), 69-84.

Mitchell, J. y Muckosy, P. (2008). A misguided quest: Community-based tourism in Latin America. Londres: ODI.

Organización Mundial del Turismo. (2004). Turismo y atenuación de la pobreza. Recomendaciones para la acción. Madrid: Autor.

Organización Mundial del Turismo. (2006). Reducción de la pobreza por medio del turismo. Una compilación de buenas prácticas. Madrid: Autor.

Pawson, S., Arcy, P. D. y Richardson, S. (2016). The value of community-based tourism in Banteay Chhmar, Cambodia. Tourism Geographies, 19(3), 378-397.

Ruiz-Ballesteros, E. (2017a). Presentación. Claves del turismo de base local. Gazeta de Antropología, 33(1).

Ruiz-Ballesteros, E. (2017b). Socio-ecological balance in community-based tourism experiences: a Research proposal. En R. Butler (Ed.). Tourism and resilience (pp. 41452). Wallingford: $C A B I$.

Ruiz-Ballesteros, E. y Cáceres-Feria, R. (2016). Community-building and amenity migration in community-based tourism development. An approach from southwest Spain. Tourism Management, 54, 513-523.

Ruiz-Ballesteros, E., Hernández, M., Coca, A., Cantero, P. y Del Campo, A. (2008). Turismo comunitario en Ecuador: Comprendiendo el community-based desde la comunidad. Pasos. Revista de Turismo y Patrimonio Cultural, 6(3), 399-418.

Sakata, H. y Prideaux, B. (2013). An alternative approach to community-based ecotourism: A bottom-up locally initiated non-monetised project in Papua New Guinea. Journal of Sustainable Tourism, 21(6), 880-899.

SNV y OMT. (2010). Manual on tourism and poverty alleviation. Practical steps for destinations. Madrid: Organización Mundial del Turismo.

Timothy, D. J. y White, K. (1999). Community-based ecotourism development on the periphery of Belize. Current Issues in Tourism, 2(2-3), 226-242.

Tolkach, D. y King, B. (2015). Strengthening Community-Based Tourism in a new resource-based island nation: Why and how? Tourism Management, 48, 386-398.

Zapata, M.J, Hall, C. M., Lindo, P., Vanderschaeghe, M. (2011). Can community-based tourism contribute to development and poverty alleviation? Lessons from Nicaragua. Current Issues in Tourism, 4(8), 1-25. 